\title{
Progressive Ependymoma
}

National Cancer Institute

\section{Source}

National Cancer Institute. Progressive Ependymoma. NCI Thesaurus. Code C147112.

Ependymoma that is increasing in scope or severity. 